\title{
Oscillations in the spectrum of nonlinear Thomson-backscattered radiation
}

\author{
C. A. Brau \\ Department of Physics and Astronomy, Vanderbilt University, Nashville, Tennessee 37235, USA
}

(Received 14 October 2003; published 9 February 2004)

\begin{abstract}
When an electron beam collides with a high-intensity laser beam, the spectrum of the nonlinear Thomson scattering in the backward direction shows strong oscillations like those in the spectrum of an optical klystron. Laser gain on the backward Thomson scattering is estimated using the Madey theorem, and the results suggest that Thomson-backscatter free-electron lasers are possible at wavelengths extending to the far uv using a terawatt laser beam from a chirped-pulse amplifier and a highbrightness electron beam from a needle cathode.
\end{abstract}

DOI: 10.1103/PhysRevSTAB.7.020701

PACS numbers: 41.60.Ap, 52.59.-f

\section{INTRODUCTION}

Thomson scattering is the radiation emitted when a charged particle oscillates in the electromagnetic field of an incident optical wave. When the optical field is small, the particle motions are just one-dimensional oscillations in the electric field of the incident wave. The scattered intensity is linear in the incident intensity and appears at the same frequency. If the electrons are moving toward the incident optical beam, the radiation is Doppler shifted to higher frequencies. This has proved to be an important source of $\mathrm{x}$ rays and gamma rays [13]. When the optical field is sufficiently intense, the particle motions are relativistic and the radiation is nonlinear. In this case, the scattered radiation includes harmonics and the spectrum is broadened by Doppler shifts caused by the (classical) recoil of the particle in the optical field. The development in recent years of high-power (terawatt) pulsed lasers has made it possible to observe both the harmonics and the Dopplerbroadened radiation scattered from electrons in dilute plasmas [4].

It has also been proposed to use nonlinear Thomson scattering to make a tabletop free-electron laser (FEL). The first such proposal was put forward in 1968 by Pantell, Soncini, and Puthoff [5], who predicted that usable gain could be achieved in the far-infrared region using the technology available at that time. Since then, there have been several proposals to develop a Thomson FEL for shorter wavelengths [6-9]. At the present time, several very large projects are underway in Germany and the United States to develop conventional undulator freeelectron lasers for the far-uv and x-ray parts of the spectrum using $\mathrm{GeV}$ accelerators [10], but the idea of a tabletop free-electron laser has not been pursued because of the limitations of available technology. Recent developments in both lasers (chirped-pulse amplifiers [11]) and electron beams (needle photocathodes [12]) may change this situation, however. A compact and relatively inexpensive alternative to conventional approaches for a uv or $\mathrm{x}$-ray FEL would be most useful.

\section{THOMSON SCATTERING}

In the present paper, we analyze nonlinear Thomson backscatter from an electron beam. Previous analyses have considered the case when the incident radiation has uniform intensity during the pulse [5-9,13,14], although recently computer simulations of nonlinear Thomson scattering from plasmas have been done for nonuniform laser pulses [15]. In the following, we consider the case of a smoothly varying pulse and specialize to a Gaussian pulse shape as an example. We find that the spectrum of the backscattered radiation has interesting structure similar to that observed from the so-called optical-klystron free-electron lasers [16]. This has a pronounced effect on the optical gain of the backscattered radiation.

The angular spectral fluence of radiation from a moving charge is given by the formula (SI units are used throughout) $[17,18]$

$$
\frac{d^{2} \mathcal{W}}{d \omega d \Omega}=\frac{\mu_{0} c \omega^{2} q^{2}}{16 \pi^{3}}\left|\int_{-\infty}^{\infty} d t e^{i \omega(t-\hat{\mathbf{n}} \cdot \mathbf{r} / c)} \hat{\mathbf{n}} \times \boldsymbol{\beta}\right|^{2},
$$

where $\mu_{0}$ is the permeability of free space, $c$ the speed of light, $\omega$ the angular frequency, $q$ the charge on the particle (negative for electrons), $t$ the time, $\mathbf{r}$ the position of the particle, $c \boldsymbol{\beta}=d \mathbf{r} / d t$ the velocity of the particle, and $\hat{\mathbf{n}}$ a unit vector in the direction of the observer. For a linearly polarized incident plane wave with the wave vector $\mathbf{k}_{0}$ and polarization $\hat{\mathbf{e}}_{0}$, we define $\hat{k}^{\alpha}=\left(1, \hat{\mathbf{k}}_{0}\right)$, where $\hat{\mathbf{k}}_{0}=\mathbf{k}_{0} / k_{0}$, and $\hat{\boldsymbol{e}}^{\alpha}=\left(0, \hat{\mathbf{e}}_{0}\right)$. The 4 -vector potential in the Coulomb gauge is then

$$
A^{\alpha}(\mathbf{r}, t)=A(\xi) \hat{e}^{\alpha},
$$

where

$$
\xi=c t-\hat{\mathbf{k}}_{0} \cdot \mathbf{r}=\hat{k}^{\alpha} r_{\alpha},
$$

and $r^{\alpha}=(c t, \mathbf{r})$ is the 4 -vector position. We consider a particle initially traveling parallel to the incident wave in the direction $\hat{\mathbf{k}}_{0}$, as shown in Fig. 1. The electron has the initial velocity $\beta_{0} c$ and momentum $\mathbf{p}_{0}=\beta_{0} \gamma_{0} m c \hat{\mathbf{k}}_{0}$, 


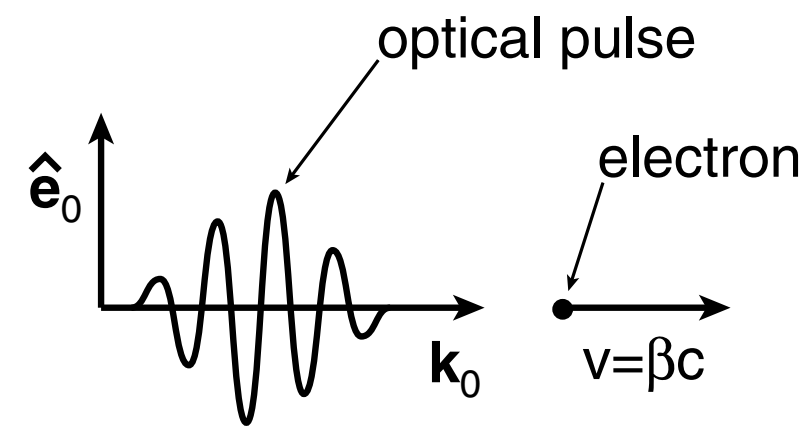

FIG. 1. Geometry of optical pulse incident on an electron traveling in the same direction.

where $m$ is the particle rest mass and $\gamma_{0}=1 / \sqrt{1-\beta_{0}^{2}}$, so for head-on collision of the optical pulse with an electron traveling in the opposite direction we take $\beta_{0}<0$. The equation of motion for the 4 -vector momentum $p^{\alpha}$ is

$$
\frac{d p^{\alpha}}{d \tau}=\frac{q}{m}\left(\partial^{\alpha} A^{\beta}-\partial^{\beta} A^{\alpha}\right) p_{\beta}
$$

If we substitute the potential (2) into the equation of motion and recognize that $\partial^{\alpha} A=\hat{k}^{\alpha} d A / d \xi$, we find that

$$
\frac{d p^{\alpha}}{d \tau}=\frac{q}{m} \frac{d A}{d \xi}\left(p_{\beta} \hat{e}^{\beta} \hat{k}^{\alpha}-p_{\beta} \hat{k}^{\beta} \hat{e}^{\alpha}\right)
$$

If we take the inner product with $\hat{k}_{\alpha}$, the right-hand side vanishes and we obtain the useful result

$$
\frac{d \xi}{d \tau}=\frac{d \hat{k}_{\alpha} r^{\alpha}}{d \tau}=\frac{\hat{k}_{\alpha} p^{\alpha}}{m}=\frac{\mathcal{E}_{0}}{m c}
$$

for some constant $\mathcal{E}_{0}$. That is, the parameter $\xi$ is just a constant times the proper time $\tau$. If we evaluate the constant before the pulse arrives, we find that

$$
\mathcal{E}_{0}=c \hat{k}_{\alpha} p^{\alpha}=\gamma_{0} m c^{2}-\gamma_{0} \beta_{0} m c^{2}=\gamma_{0}\left(1-\beta_{0}\right) m c^{2} .
$$

Since the canonical momentum in the transverse direction is conserved, it follows immediately that

$$
\mathbf{p} \cdot \hat{\mathbf{e}}_{0}+q A(\xi)=0=-p^{\alpha} \hat{e}_{\alpha}+q A(\xi) .
$$

If we substitute (6)-(8) into (5) and take the component in the longitudinal direction, we obtain the equation of motion

$$
\frac{d}{d \xi}\left(\hat{\mathbf{k}}_{0} \cdot \mathbf{p}\right)=\frac{q^{2}}{m c \gamma_{0}\left(1-\beta_{0}\right)} A \frac{d A}{d \xi} .
$$

Integrating once and evaluating the constant of integration before the pulse arrives, we get

$$
\hat{\mathbf{k}}_{0} \cdot \mathbf{p}=\frac{q^{2}}{m c \gamma_{0}\left(1-\beta_{0}\right)} \frac{A^{2}}{2}+\gamma_{0} \beta_{0} m c=\frac{1}{m} \hat{\mathbf{k}}_{0} \cdot \frac{d \mathbf{r}}{d \tau} .
$$

Using (6) to change the independent variable to $\xi$ and integrating a second time, we find that the longitudinal position is $[19,20]$

$$
\hat{\mathbf{k}}_{0} \cdot \mathbf{r}=\frac{\beta_{0}}{1-\beta_{0}} \xi+\frac{1}{2} \frac{1+\beta_{0}}{1-\beta_{0}} \int_{0}^{\xi} a^{2}(\xi) d \xi
$$

where we have introduced the dimensionless vector potential $a(\xi)=q A(\xi) / m c$. When we substitute these results into (1), we find that the angular spectral fluence in the backscattered direction $\left(\hat{\mathbf{n}}=-\hat{\mathbf{k}}_{0}\right)$ is

$$
\frac{d^{2} \mathcal{W}}{d \omega d \Omega}=\frac{\mu_{0} q^{2} \omega^{2}}{16 \pi^{3} c} \frac{1+\beta_{0}}{1-\beta_{0}}\left|\int_{-\infty}^{\infty} e^{i(\omega / c)\left[\left(1+\beta_{0}\right) /\left(1-\beta_{0}\right)\right]\left[\xi+\int_{0}^{\xi} a^{2}(\xi) d \xi\right]} a(\xi) d \xi\right|^{2} .
$$

We are interested in the case when the incident pulse includes many oscillations under a slowly varying envelope, so we write

$$
a(\xi)=\bar{a}(z) \cos \left(\nu_{0} z\right)
$$

where

$$
z=\xi / \xi_{0}
$$

and

$$
\nu_{0}=\frac{\omega_{0} \xi_{0}}{c} \gg 1,
$$

in which $\omega_{0}$ is the frequency of the incident pulse and $\xi_{0}$ the pulse length. In the limit $\nu_{0} \gg 1$, we can develop an asymptotic series for the integral in the exponent of (12) by integrating repeatedly by parts. For the first term we get

$$
\begin{aligned}
\frac{\omega}{c} \frac{1+\beta_{0}}{1-\beta_{0}}\left[\xi+\int_{0}^{\xi} a^{2}(\xi) d \xi\right] & =\nu_{0} w \frac{1+\beta_{0}}{1-\beta_{0}}\left\{z+\frac{1}{2} \int_{0}^{z} \bar{a}^{2}(z)\left[1+\cos \left(2 \nu_{0} z\right)\right] d z\right\} \\
& \approx \nu_{0} w \frac{1+\beta_{0}}{1-\beta_{0}} Z(z)+\frac{1}{4} w \frac{1+\beta_{0}}{1-\beta_{0}} \bar{a}^{2}(z) \sin \left(2 \nu_{0} z\right)+O\left(1 / \nu_{0}\right),
\end{aligned}
$$

where the dimensionless frequency is 


$$
w=\omega / \omega_{0}
$$

and

$$
Z(z)=z+\frac{1}{2} \int_{0}^{z} \bar{a}^{2}(z) d z
$$

The angular spectral fluence is then

$$
\frac{d^{2} W}{d \Omega d \omega}=\frac{\mu_{0} c q^{2}}{16 \pi^{3}} \frac{1+\beta_{0}}{1-\beta_{0}} \nu_{0}^{2} w^{2}\left|\int_{-\infty}^{\infty} d z \bar{a}(z) \cos \left(\nu_{0} z\right) e^{i \nu_{0} w\left(1+\beta_{0} / 1-\beta_{0}\right) Z(z)} e^{i(w / 4)\left[\left(1+\beta_{0}\right) /\left(1-\beta_{0}\right)\right] \bar{a}^{2}(z) \sin \left(2 \nu_{0} z\right)}\right|^{2} .
$$

Using the Bessel function expansion

$$
e^{i \alpha \sin (\theta)}=\sum_{n=-\infty}^{\infty} J_{n}(\alpha) e^{i n \theta}
$$

for the last factor and rearranging the sums using $J_{-n}=(-1)^{n} J_{n}$, we get

$$
\frac{d^{2} \mathcal{W}}{d \Omega d \omega}=\frac{\mu_{0} c q^{2}}{64 \pi^{3}} \frac{1+\beta_{0}}{1-\beta_{0}} \nu_{0}^{2} w^{2}\left|\sum_{n=-\infty}^{\infty} \int_{-\infty}^{\infty} d z \bar{a}(z) K_{n}\left[\frac{w}{4} \frac{1+\beta_{0}}{1-\beta_{0}} \bar{a}^{2}(z)\right] e^{i \nu_{0}\left\{w\left[\left(1+\beta_{0}\right) /\left(1-\beta_{0}\right)\right] Z(z)-(2 n-1) z\right\}}\right|^{2},
$$

where we define

$$
K_{n}(\alpha)=(-1)^{n}\left[J_{n}(\alpha)-J_{n-1}(\alpha)\right] .
$$

But $Z(z)$ is a monotonically increasing function of $z$, so the exponential in (21) oscillates rapidly unless there is a resonance between the first and second terms. This can occur only for $n \geq 1$, so we can ignore the rest of the terms in the sum. For convenience in the following we assume that the incident pulse is symmetric, so that $\bar{a}(z)$ is an even function of $z$. The angular spectral fluence is then

$$
\frac{d^{2} W}{d \Omega d \omega}=\frac{\mu_{0} c q^{2}}{16 \pi^{3}} \frac{1+\beta_{0}}{1-\beta_{0}} \nu_{0}^{2} w^{2}\left[\sum_{n=1}^{\infty} I_{n}(w)\right]^{2}
$$

where

$$
I_{n}(w)=\int_{0}^{\infty} d z \bar{a}(z) K_{n}\left[\frac{w}{4} \frac{1+\beta_{0}}{1-\beta_{0}} \bar{a}^{2}(z)\right] \cos \left\{\nu_{0}\left[w \frac{1+\beta_{0}}{1-\beta_{0}} Z(z)-(2 n-1) z\right]\right\}
$$

For the specific case of a Gaussian pulse, the envelope of the amplitude is

$$
\bar{a}(z)=a_{0} e^{-z^{2}}
$$

and

$$
Z(z)=z+\sqrt{\frac{\pi}{2}} \frac{a_{0}^{2}}{4} \operatorname{erf}(\sqrt{2} z)
$$

where $\operatorname{erf}(x)$ is the error function.

An example of the spectrum obtained using the asymptotic approximation is shown in Fig. 2. The laser parameters used in the computations correspond to what can be achieved with a tabletop chirped-pulse amplifier: a total energy $\mathcal{U}=5 \mathrm{~J}$ in a 10 -ps pulse $\left(\xi_{0} \approx 3.0 \mathrm{~mm}\right)$ at a wavelength $\lambda_{0}=1.054 \mu \mathrm{m} \quad\left(\nu_{0} \approx 1.8 \times 10^{4}\right)$, focused to a $53-\mu \mathrm{m}$ diameter spot $\left(a_{0} \approx 0.17\right)$. The electron velocity used in the computations corresponds to $100 \mathrm{keV}$ $\left(\beta_{0}=-0.55\right)$, which can achieved with a tabletop de gun. The results obtained with the asymptotic approximation are indistinguishable from those obtained by numerical integration of the exact result (12). As shown in Fig. 2, the spectrum of radiation on the fundamental extends from the nominal (twice-Doppler-shifted) wavelength $\lambda \approx$ $308 \mathrm{~nm}$ out to $\lambda \approx 312 \mathrm{~nm}$, due to the (classical) recoil from the incident laser pulse. The oscillatory structure in the spectrum is reminiscent of the spontaneous emission from an optical-klystron free-electron laser and arises in the same way [16]. Near the beginning of the interaction the electron emits radiation at the frequency $\omega$, but as the intensity of the incident pump pulse increases, the electron is accelerated in the direction of the incident pulse and changes frequency (Doppler shift) as it is pushed backward. When the pump intensity decreases later in the pulse the electron radiates at the frequency $\omega$ once again. However, this radiation is phase shifted relative to the earlier radiation and interferes with it constructively or destructively depending on the precise value of the phase shift.

The analogy with the radiation from an optical-klystron FEL suggests that the optical gain on this backscattered radiation might be enhanced by the oscillatory structure. We can estimate the small-signal gain by means of Madey's theorem, which states that in the limit of small gain, the small-signal gain is related to the spontaneous emission by the formula $[21,22]$

$$
G-1=-\frac{8 \pi^{3} J_{e}}{m q \omega^{2}} \frac{d}{d \gamma_{0}}\left(\frac{d^{2} W}{d \omega d \Omega}\right)
$$


where $J_{e}$ is the current density $\left(J_{e}>0\right.$ for negatively charged electrons traveling to the left). But $d^{2} \mathcal{W} / d \Omega d \omega$ depends on $\gamma_{0}$ only through the factor $\left(1+\beta_{0}\right) /\left(1-\beta_{0}\right)$. This appears in several places in (24) and (25), but the strongest dependence is due to its appearance in the cosine, where it is multiplied by $\nu_{0} \gg 1$. Ignoring all but the strongest dependence, we get

$$
G-1=-\frac{2 \mu_{0} c q J_{e}}{m \omega_{0}^{2} \gamma_{0} \beta_{0}} \nu_{0}^{2}\left(\frac{1+\beta_{0}}{1-\beta_{0}}\right)^{2}\left[\sum_{n=1}^{\infty} I_{n}(w)\right]\left[\sum_{n=1}^{\infty} I_{n}^{\prime}(w)\right]
$$

where

$$
I_{n}^{\prime}(w)=-\nu_{0} w \int_{0}^{\infty} d z \bar{a}(z) K_{n}\left[\frac{w}{4} \frac{1+\beta_{0}}{1-\beta_{0}} \bar{a}^{2}(z)\right] Z(z) \sin \left\{\nu_{0}\left[w \frac{1+\beta_{0}}{1-\beta_{0}} Z(z)-(2 n-1) z\right]\right\}
$$

The gain calculated from these formulas for a Gaussian pulse is shown in Fig. 3. The parameters used for this figure are the same as those used for Fig. 2, but with an electron-beam total current $I_{e}=100 \mathrm{~mA}$ focused to a $60.2-\mu \mathrm{m}$ diameter spot $\left(J_{e} \approx 1.0 \times 10^{9} \mathrm{~A} / \mathrm{m}^{2}\right)$, which could be achieved from a needle photocathode [12]. As shown in Fig. 3, the gain exhibits large positive and negative oscillations, with a peak gain $G-1-10$. It is of interest to note in passing that $q J_{e} / \beta_{0}=q^{2} c n_{e}>0$, where $n_{e}$ is the electron density. Thus, the gain remains finite even when the electron beam is stationary $\left(\beta_{0} \rightarrow 0\right)$, as must be true since we can view the gain from an electron beam in the reference frame where the beam is stationary. This suggests that stimulated Thomson backscatter should be observed from a sufficiently dense, cold plasma, although in this case the wavelength where the gain appears is longer than the incident wavelength due to the recoil of the electrons in the laboratory frame.

\section{LONG PULSES}

A few calculations using (28) are sufficient to show that the gain is larger for longer pulses, even if the total energy is held constant. For very long pulses $\left(\nu_{0} \gg 1\right)$, the integrals (24) and (29) are difficult to evaluate numerically, but they can be conveniently approximated using the method of stationary phase [23]. At a given frequency, the dominant contribution to the integrals (24) and (29) comes from the point in the pulse where the local emission frequency is Doppler shifted to the frequency we are examining. This is the point of stationary phase. If we call the stationary-phase point $z_{0}$ and expand about this point, then the argument of the sine and the cosine is

$$
\begin{aligned}
\nu_{0}\left[w \frac{1+\beta_{0}}{1-\beta_{0}} Z(z)-(2 n-1) z\right] \approx & \nu_{0}\left[w \frac{1+\beta_{0}}{1-\beta_{0}} Z\left(z_{0}\right)-(2 n-1) z_{0}\right]+\nu_{0}\left[w \frac{1+\beta_{0}}{1-\beta_{0}} Z^{\prime}\left(z_{0}\right)-(2 n-1)\right]\left(z-z_{0}\right) \\
& +\frac{\nu_{0} w}{2} \frac{1+\beta_{0}}{1-\beta_{0}} Z^{\prime \prime}\left(z_{0}\right)\left(z-z_{0}\right)^{2} .
\end{aligned}
$$

At the point of stationary phase the derivative of the phase vanishes, so the stationary-phase point $z_{0}$ satisfies the condition

$$
w \frac{1+\beta_{0}}{1-\beta_{0}}\left[1+\frac{1}{2} \bar{a}^{2}\left(z_{0}\right)\right]=2 n-1 .
$$

This is the resonance condition, and it has solutions for frequencies in the range

$$
(2 n-1) \frac{1-\beta_{0}}{1+\beta_{0}} \frac{1}{1+\frac{1}{2} \bar{a}^{2}(0)}<w<(2 n-1) \frac{1-\beta_{0}}{1+\beta_{0}} .
$$

The integrals (24) and (29) are then

$$
\begin{aligned}
& I_{n}(w) \approx \bar{a}\left(z_{0}\right) K_{n}\left[\frac{w}{4} \frac{1+\beta_{0}}{1-\beta_{0}} \bar{a}^{2}\left(z_{0}\right)\right] \int_{0}^{\infty} d z \cos \left\{\nu_{0}\left[w \frac{1+\beta_{0}}{1-\beta_{0}} Z\left(z_{0}\right)-(2 n-1) z_{0}+\frac{w}{2} \frac{1+\beta_{0}}{1-\beta_{0}} Z^{\prime \prime}\left(z_{0}\right)\left(z-z_{0}\right)^{2}\right]\right\}, \\
& I_{n}^{\prime}(w) \approx-\bar{a}\left(z_{0}\right) K_{n}\left[\frac{w}{4} \frac{1+\beta_{0}}{1-\beta_{0}} \bar{a}^{2}\left(z_{0}\right)\right] \nu_{0} w Z\left(z_{0}\right) \int_{0}^{\infty} d z \sin \left\{\nu _ { 0 } \left[w \frac{1+\beta_{0}}{1-\beta_{0}} Z\left(z_{0}\right)-(2 n-1) z_{0}\right.\right. \\
& \left.\left.+\frac{w}{2} \frac{1+\beta_{0}}{1-\beta_{0}} Z^{\prime \prime}\left(z_{0}\right)\left(z-z_{0}\right)^{2}\right]\right\}
\end{aligned}
$$

Provided that $z_{0}$ is not too close to the origin, we can extend the integral to $-\infty$. If we assume that $Z^{\prime \prime}\left(z_{0}\right)=-\left|Z^{\prime \prime}\left(z_{0}\right)\right|$ 


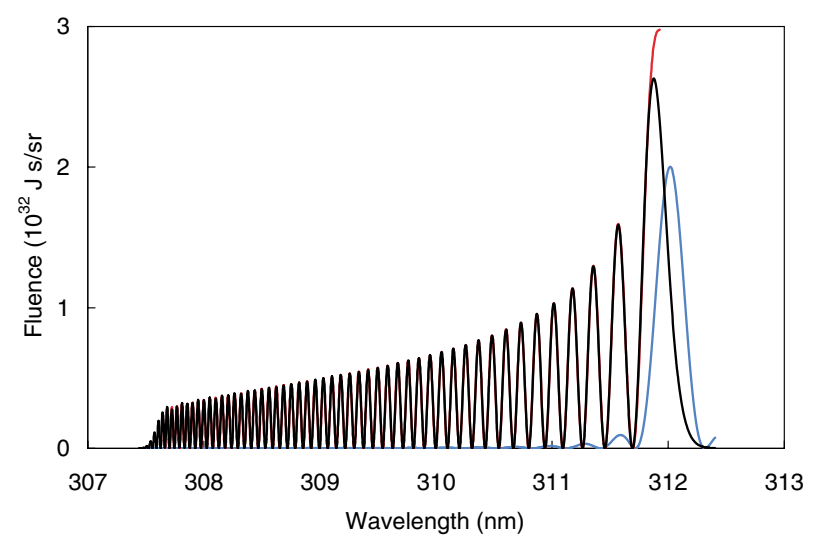

FIG. 2. (Color) Angular spectral fluence versus wavelength (fundamental only). The asymptotic approximation is shown as a black line and the stationary-phase approximation as a red line extending upward from the main peak. The blue line (shifted slightly to the right of the main peak) shows the fluence for the equivalent square pulse.

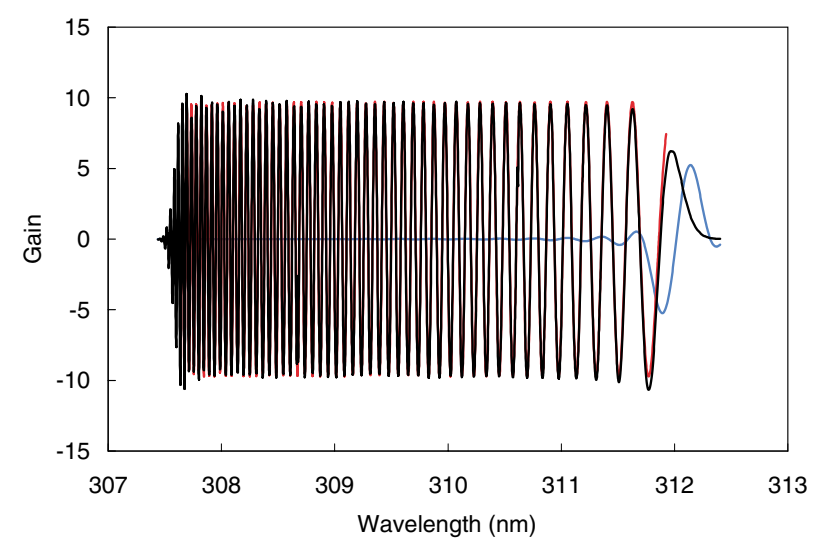

FIG. 3. (Color) Gain versus wavelength (fundamental only). The asymptotic approximation is shown as a black line and the stationary-phase approximation as a red line extending upward from the first peak. The blue line, shifted slightly to the right of the first peak, shows the gain for the equivalent square pulse.

[that is, $\bar{a}(z)$ is a monotonically decreasing function of $z$ for $z>0$ ], use trigonometric identities for $\cos (a-b), \sin (a-$ $b), \sin a+\cos a$, and $\sin a-\cos a$, and the definite integral

$$
\int_{-\infty}^{\infty} \cos x^{2} d x=\sqrt{\frac{\pi}{2}}
$$

we get

$$
\begin{gathered}
I_{n}(w)=\sqrt{\frac{2 \pi}{\nu_{0} w\left|Z^{\prime \prime}\left(z_{0}\right)\right|} \frac{1-\beta_{0}}{1+\beta_{0}}} \bar{a}\left(z_{0}\right) K_{n}\left[\frac{w}{4} \frac{1+\beta_{0}}{1-\beta_{0}} \bar{a}^{2}\left(z_{0}\right)\right] \cos \theta, \\
I_{n}^{\prime}(w)=-\sqrt{\frac{2 \pi}{\nu_{0} w\left|Z^{\prime \prime}\left(z_{0}\right)\right|} \frac{1-\beta_{0}}{1+\beta_{0}}} \bar{a}\left(z_{0}\right) K_{n}\left[\frac{w}{4} \frac{1+\beta_{0}}{1-\beta_{0}} \bar{a}^{2}\left(z_{0}\right)\right] \nu_{0} w Z\left(z_{0}\right) \sin \theta,
\end{gathered}
$$

where the phase of the spectral oscillations is

$$
\theta=\nu_{0}\left[w \frac{1+\beta_{0}}{1-\beta_{0}} Z\left(z_{0}\right)-(2 n-1) z_{0}\right]-\frac{\pi}{4}
$$

If we ignore all harmonics but the fundamental, the angular spectral fluence is

$$
\frac{d^{2} \mathcal{W}}{d \omega d \Omega}=\frac{\mu_{0} c q^{2}}{8 \pi^{2}} \frac{\nu_{0} w \bar{a}^{2}\left(z_{0}\right)}{\left|Z^{\prime \prime}\left(z_{0}\right)\right|} K_{1}^{2}\left[\frac{w}{4} \frac{1+\beta_{0}}{1-\beta_{0}} \bar{a}^{2}\left(z_{0}\right)\right] \cos ^{2}(\theta)
$$

and the gain is

$$
G-1=\frac{2 \pi \nu_{0}^{2} \mu_{0} c q J_{e}}{m \omega_{0}^{2} \gamma_{0} \beta_{0}} \frac{1+\beta_{0}}{1-\beta_{0}} \bar{a}^{2}\left(z_{0}\right) K_{1}^{2}\left[\frac{w}{4} \frac{1+\beta_{0}}{1-\beta_{0}} \bar{a}^{2}\left(z_{0}\right)\right] \frac{Z\left(z_{0}\right)}{\left|Z^{\prime \prime}\left(z_{0}\right)\right|} \sin (2 \theta) .
$$

The condition for the stationary-phase approximation to be valid is that the width of the resonance be small compared with the value of $z_{0}$ when the resonance occurs near the origin $\left(z_{0} \ll 1\right)$ or small compared with unity (the scale length for variations of the pulse amplitude) otherwise. That is, we require

$$
\frac{\nu_{0} w}{2} \frac{1+\beta_{0}}{1-\beta_{0}}\left|Z^{\prime \prime}\left(z_{0}\right)\right| \min \left(z_{0}^{2}, 1\right) \gg 1
$$

This will be true except near $z_{0}=0$ or for $z_{0} \gg 1$, where $Z^{\prime \prime}\left(z_{0}\right)$ becomes small. Therefore, the stationary-phase approximation should be valid except near the endpoints of the spectrum of each individual harmonic.

For the specific case of a Gaussian pulse, as discussed earlier,

$$
Z^{\prime}(z)=1+\frac{1}{2} a_{0}^{2} e^{-2 z^{2}}
$$


and

$$
Z^{\prime \prime}(z)=-2 a_{0}^{2} z e^{-2 z^{2}}
$$

The stationary-phase point is then

$$
z_{0}=\sqrt{\ln \sqrt{a_{0}^{2} /\left(\frac{4 n-2}{w} \frac{1-\beta_{0}}{1+\beta_{0}}-2\right)}}
$$

The stationary-phase approximation for a Gaussian pulse is compared with the asymptotic approximation in Figs. 2 and 3, where the asymptotic approximation is shown as a black line and the stationary-phase approximation as a red line. As shown there, the stationary-phase approximation works quite well except near the end points of the spectrum, as expected.

Using (40), it is now a simple matter to estimate the gain at the first (longest wavelength) full peak in the spectrum, which we chose because it is the broadest full peak. This peak occurs at $\theta=3 \pi / 4$, where $\sin 2 \theta=-1$. Since the first full peak occurs for $z_{0} \ll 1$, we may use the approximation

$$
\frac{Z\left(z_{0}\right)}{\left|Z^{\prime \prime}\left(z_{0}\right)\right|} \approx \frac{1+\frac{1}{2} a_{0}^{2}}{2 a_{0}^{2}} .
$$

The value of the gain at the peak is therefore

$$
G_{\max }-1=\frac{\pi \nu_{0}^{2} \mu_{0} c q J_{e}}{m \omega_{0}^{2} \gamma_{0} \beta_{0}} \frac{1+\beta_{0}}{1-\beta_{0}}\left(1+\frac{1}{2} a_{0}^{2}\right) K_{1}^{2}\left[\frac{w}{4} \frac{1+\beta_{0}}{1-\beta_{0}} a_{0}^{2}\right] .
$$

Using these same approximations in (38) we find that the first full gain peak occurs at

$$
z_{0}=\left(\frac{3 \pi}{2} \frac{1+\frac{1}{2} a_{0}^{2}}{\nu_{0} a_{0}^{2}}\right)^{1 / 3},
$$

which is small for long pulses $\left(\nu_{0} \gg 1\right)$. The condition for the validity of the stationary-phase approximation at the first gain peak is then

$$
\nu_{0} w \frac{1+\beta_{0}}{1-\beta_{0}} a_{0}^{2} z_{0}^{3}=\frac{3 \pi}{2} w \frac{1+\beta_{0}}{1-\beta_{0}}\left(1+\frac{1}{2} a_{0}^{2}\right) \gg 1 .
$$

But near the first gain peak, we see from the resonance condition (31) that $w\left(1+\frac{1}{2} a_{0}^{2}\right)\left(1+\beta_{0}\right) /\left(1-\beta_{0}\right) \approx 1$, so this leaves us with the condition $3 \pi / 2 \gg 1$. As we see in Fig. 3, this is good enough for our purposes since the stationary-phase approximation is close to the asymptotic approximation at the first full peak of the gain.

\section{RESULTS}

We can use the simple formula (46) to study the scaling of the gain with the pump laser energy, the pulse length, and the wavelength of the laser output. To do this we have to make some assumptions about the geometry of the interaction, which is shown in Fig. 4. For a pump laser with power $\mathcal{P}$ in the lowest Gaussian mode focused to a Rayleigh range $z_{R}$, the dimensionless vector potential on axis at the focus is

$$
a_{0}^{2}=\frac{\mu_{0} q^{2} \lambda_{0} \mathcal{P}}{\pi^{2} m^{2} c^{3} z_{R}}
$$

where $\lambda_{0}=2 \pi c / \omega_{0}$ is the pump wavelength. For an electron traveling at the velocity $\beta_{0} c$, the distance traveled during the interaction with a copropagating optical pulse of total duration $\approx 2 \Delta t$ is $2\left|\beta_{0}\right| c \Delta t /\left(1-\beta_{0}\right)$. To keep the effects of diffraction of the pump laser small, we make the Rayleigh range equal to this distance and set

$$
z_{R}=\frac{2\left|\beta_{0}\right| c \Delta t}{1-\beta_{0}}
$$

The dimensionless vector potential $a_{0}$ at the focus is then found from

$$
\nu_{0} a_{0}^{2}=\sqrt{\frac{2}{\pi}} \frac{\mu_{0} q^{2}}{\pi m^{2} c^{3}} \frac{1-\beta_{0}}{\left|\beta_{0}\right|} \frac{U}{\Delta t},
$$

where $\mathcal{U}$ is the energy in the Gaussian laser pulse. The degree to which the electron beam can be focused is determined by the spread of the beam in its transverse phase space. In terms of the effective [24] emittance $\varepsilon$ of the electron beam (roughly the total area of the beam in transverse phase space divided by $\pi, 4$ times larger than the rms emittance [24]) and the depth of focus $z_{e}$ (equivalent to the Rayleigh range of the pump laser), the area of the focused electron beam is $\pi \varepsilon z_{e}$ [25]. The electron beam can be focused somewhat more tightly than the laser beam, since the interaction is important only between the resonant points, so we take $z_{e}=z_{0} z_{R}$. Since the emittance generally increases with the total current, a better invariant measure of the electron-beam quality is the normalized brightness $B_{N} \approx 2 I_{e} / \pi^{2} \gamma_{0}^{2} \beta_{0}^{2} \varepsilon^{2}$. In terms of the normalized brightness and the total current $I_{e}$, the current density at the focus is then

$$
J_{e}=\frac{I}{\pi \varepsilon z_{e}} \approx \sqrt{B_{N} I_{e}}\left|\frac{1}{48 \pi^{5 / 2}} \frac{\mu_{0} q^{2}}{m^{2} c^{6}} \frac{\gamma_{0}^{3}\left(1-\beta_{0}\right)^{4}}{\beta_{0}} \frac{U}{\Delta t^{4}}\right|^{1 / 3}
$$

for $a_{0}^{2} \ll 1$, which will typically be the case for long

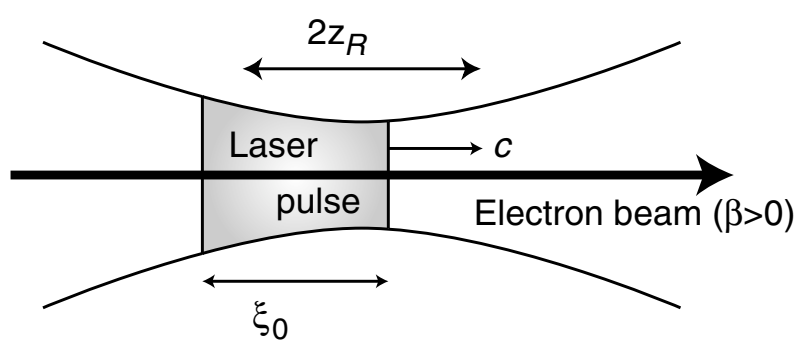

FIG. 4. Geometry of the electron beam and laser beam in the interaction region. 
pulses. Combining these formulas, using the fact that $K_{1}^{2}(x) \approx 1$ for $x \ll 1$, we find that for $a_{0}^{2} \ll 1$, which is typical of long pulses, the peak gain is given by the simple formula

$$
G_{\max }-1 \approx \frac{1+\beta_{0}}{1-\beta_{0}} \sqrt{B_{N} I_{e}}\left|\frac{\sqrt{\pi}}{48} \frac{\mu_{0}^{4} q^{5}}{m^{5} c^{3}}\left(\frac{1-\beta_{0}}{\beta_{0}}\right)^{4} \mathcal{U} \Delta t^{2}\right|^{1 / 3} .
$$

This shows that the gain increases with laser pulse energy and, more strongly, pulse length. Some results are shown in Fig. 5 for the parameters used previously $\left(I_{e}=\right.$ $100 \mathrm{~mA}, \beta_{0} \approx-0.55, B_{N}=10^{13} \mathrm{~A} / \mathrm{m}^{2} \mathrm{sr}$ ) but with a more powerful laser $(\mathcal{U}=500 \mathrm{~J})$ such as the petawatt upgrade of the Vulcan laser system [26]. The pulse length $\Delta t$ is varied while keeping the total laser pulse energy $\mathcal{U}$ constant. As shown in Fig. 5, the gain can be quite large even for a relatively small current.

Several caveats are important, however. In the first place, the Madey theorem is valid only in the case when the gain is small, $G-1 \ll 1$, which is clearly not the case in Fig. 5, or in Fig. 3, for that matter. Typically, however, the gain becomes exponentially larger when $G-1 \gg 1$, which would make the present estimates conservative. We can illustrate the importance of this effect by considering the gain in a uniform optical pulse, for in this case the exponential gain can also be calculated analytically. For a uniform pulse

$$
a=a_{0}, \quad-\xi_{L}<\xi<\xi_{L},
$$

with the effective length $2 \xi_{L}$, we can compute the spectrum and gain on the fundamental using just the first term in the sums in (23) and (28). The stationary-phase approximation cannot be used, but the asymptotic formulas (24) and (29) remain valid. For a uniform pulse, at frequencies near the resonant frequency

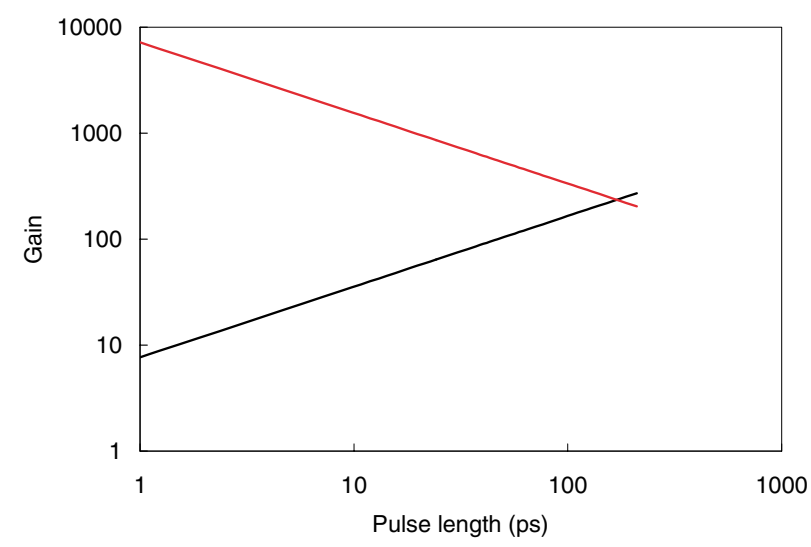

FIG. 5. (Color) Gain versus pulse length for constant pulse energy $(500 \mathrm{~J})$. The gain for an emittance-dominated electron beam is indicated by the black (lower) line, and that for a space-charge-dominated beam by the red (upper) line. The curves terminate at the quantum limit.

$$
w_{L}=\frac{1-\beta_{0}}{1+\beta_{0}} \frac{1}{1+\frac{1}{2} a_{0}^{2}}
$$

we find that

$$
I_{1}(w)=a_{0} z_{L} K_{1}\left(\frac{1}{4} \frac{a_{0}^{2}}{1+\frac{1}{2} a_{0}^{2}}\right) \frac{\sin (x)}{x},
$$

where the dimensionless effective length is

$$
z_{L}=\xi_{L} / \xi_{0}
$$

and the dimensionless departure from resonance is

$$
x=\nu_{0} z_{L} \frac{w-w_{L}}{w_{L}} .
$$

Substituting this into (23), we find that the angular spectral fluence is

$$
\frac{d^{2} \mathcal{W}}{d \omega \Omega}=\frac{\mu_{0} c q^{2}}{16 \pi^{3}} \frac{1-\beta_{0}}{1+\beta_{0}} \frac{a_{0}^{2} \nu_{0}^{2} z_{L}^{2}}{\left(1+\frac{1}{2} a_{0}^{2}\right)^{2}} K_{1}^{2}\left(\frac{1}{4} \frac{a_{0}^{2}}{1+\frac{1}{2} a_{0}^{2}}\right)\left[\frac{\sin (x)}{x}\right]^{2} .
$$

In the same way, we find that the small-signal gain is

$$
G-1=j_{e} \frac{x \sin (x) \cos (x)-\sin ^{2}(x)}{x^{3}},
$$

where

$$
j_{e}=\frac{2 \mu_{0} c q J_{e}}{m \omega_{0}^{2} \gamma_{0} \beta_{0}} \frac{1+\beta_{0}}{1-\beta_{0}} a_{0}^{2} \nu_{0}^{3} z_{L}^{3} K_{1}^{2}\left(\frac{1}{4} \frac{a_{0}^{2}}{1+\frac{1}{2} a_{0}^{2}}\right)
$$

is the dimensionless current density. The peak value of the gain profile occurs at $x=-1.303$, which is slightly below the nominal resonance.

To apply these formulas even approximately to a nonuniform pulse, we must remember that since the dimensionless vector potential varies during the pulse, only parts of the pulse are in resonance at a given frequency. We assume that the central portion of the pulse is responsible for the first gain peak and ignore the rest of the pulse. Near the center of the pulse we can use the approximation $\bar{a} \approx a_{0}\left(1-z^{2}\right)$, so from (55) we see that the shift of the resonant frequency is

$$
\frac{\Delta w}{w_{L}} \approx \frac{a_{0}^{2} z^{2}}{1+\frac{1}{2} a_{0}^{2}} .
$$

From (59) we see that the width of the resonance [the first zero of $\sin (x) / x]$ corresponds to $x=\pi$. The effective length of the pulse therefore satisfies

$$
\nu_{0}^{3} z_{L}^{3}=\pi \nu_{0}^{2} \frac{1+\frac{1}{2} a_{0}^{2}}{a_{0}^{2}}
$$

which we can substitute in (59) and (61).

These results are compared to the results for a Gaussian pulse in Figs. 2 and 3. For the parameters used for Fig. 3, the peak gain of the equivalent uniform pulse in the small-gain limit is $G_{\max }-1 \approx 5$. As shown in Fig. 3, this is about a factor of 2 smaller than the gain predicted by (46), but both estimates based on the Madey theorem 
are undoubtedly low. For the uniform pulse in the highgain limit, the gain is found to have the exponential dependence [27]

$$
G_{\exp }=\frac{1}{9} \exp \left(j_{e}^{1 / 3} \sqrt{3}\right) .
$$

Kim [28] has shown that when the signal begins from noise (self-amplified spontaneous emission), saturation is reached when $j_{e} \sim(4 \pi)^{3} \approx 2000$, which corresponds to $G_{\text {exp }} \sim e^{20} \approx 3 \times 10^{8}$. In the exponential-gain limit, the uniform-pulse gain (64) for the parameters used in Fig. 5 is $G_{\mathrm{exp}} \approx 5 \times 10^{4}$, which is much larger than the prediction from Madey's theorem. Although these results cannot be directly applied to nonuniform pulses, the gain in nonuniform pulses will certainly be much larger than predicted by Madey's theorem.

The second caveat that must be considered in using Madey's theorem to predict the gain is the fact that Madey's theorem is a classical result. Quantum effects can be ignored if the natural spreading of the electron wave packet is small compared to the wavelength in the electron rest frame [29]. Equivalently, the Compton recoil must be small compared with the wavelength. In terms of the phase shift $\delta \phi$ due to the recoil, the condition for ignoring quantum effects is

$$
\delta \phi=\frac{2 \hbar \omega_{0}}{m c^{2}} \sqrt{\frac{1-\beta_{0}}{1+\beta_{0}}} \omega_{0} \Delta t \ll 1,
$$

where $\hbar$ is Planck's constant divided by $2 \pi$ and $\Delta t$ the laser pulse length. In the low-power case illustrated in Figs. 2 and 3 , this amounts to $\delta \phi=2.64 \times 10^{-3}$, so quantum effects can be ignored. However, for longer pulses, quantum effects become a limiting factor, since they reduce the gain [30]. In Fig. 5, the curves are terminated at $200 \mathrm{ps}$, since quantum effects become important for longer pulses.

In the third place, we see in Fig. 3 that the gain is positive only over small frequency intervals. This means that the electron energy spread must be small enough to overlap just a single oscillation of the gain spectrum. For electron beams from dc accelerators, the energy spread is typically small, less than $1 \mathrm{eV}$, unless space-charge potentials across the beam are important. In the absence of space charge, the dominant effect is the spread of longitudinal momentum caused by the transverse motions of the beam. For an electron beam with emittance $\varepsilon$, the equivalent frequency spread is [25]

$$
\delta w_{\varepsilon}=\frac{\delta \omega}{\omega_{0}}=\frac{\left|\beta_{0}\right|}{\left(1+\beta_{0}\right)^{2}} \frac{\varepsilon}{\pi^{2} z_{e}}=\frac{1-\beta_{0}}{\left(1+\beta_{0}\right)^{2}} \frac{\varepsilon}{\pi^{2} z_{0} c \Delta t} .
$$

We can find the width of the gain peak in the following way. The phase $\theta$ of the spectral oscillations is given by (38). Differentiating this and using the resonance condition (31), we find that the full width of the gain peak, which corresponds to $\Delta \theta=\pi / 2$, is

$$
\frac{1}{\Delta w}=\frac{2}{\pi} \frac{d \theta}{d w}=\frac{2 \nu_{0}}{\pi} \frac{1+\beta_{0}}{1-\beta_{0}}\left(1+\frac{1}{2} a_{0}^{2}\right) z_{0} .
$$

The width of the gain peak must be larger than the frequency spread (66) caused by the electron beam, so we arrive at the criterion

$$
\frac{\delta w_{\varepsilon}}{\Delta w}=\frac{4}{\pi^{2}} \frac{\varepsilon}{\lambda_{0}} \frac{1+\frac{1}{2} a_{0}^{2}}{1+\beta_{0}} \ll 1 .
$$

We can estimate the effective emittance from the normalized brightness and the current using the formula $B_{N} \approx$ $2 I_{e} / \pi^{2} \gamma_{0}^{2} \beta_{0}^{2} \varepsilon^{2}$. For the parameters used in Fig. 3, for example $\left(I_{e}=100 \mathrm{~mA}, \quad \beta_{0} \approx-0.55, \quad B_{N}=\right.$ $10^{13} \mathrm{~A} / \mathrm{m}^{2} \mathrm{sr}$ ), we get $\varepsilon=7 \times 10^{-8} \mathrm{~m} \mathrm{rad}$. From this we find that $\delta w_{\varepsilon} / \Delta w=0.03 \ll 1$, so the emittance is not a problem.

Since the emittance generally increases with the electron-beam current, this would suggest that the current could be increased to increase the gain. However, as the electron-beam current is increased, space charge introduces two potentially deleterious effects. In the first place, the space-charge potential at the center of the beam, relative to the outer edge, causes a spread in the electron energy. For a uniform beam, the potential difference is [24]

$$
\Delta \Phi=\frac{I_{e}}{4 \pi \varepsilon_{0} c \beta_{0}} .
$$

Since the wavelength spread accompanying the electron energy difference must be smaller than the width of the gain peak, we get the criterion

$$
\frac{\delta w_{\mathrm{sc}}}{\Delta w} \ll 1
$$

For the parameters used in Fig. 3, for example, we get $\delta w_{\text {sc }} / \Delta w=7 \times 10^{-3} \ll 1$, so the space-charge potential is not a problem. In addition, it must be kept in mind that for very long pulses of high current, space-charge forces prevent the electron beam from being focused to a small spot. From the envelope equation for an electron beam with space charge, it can be shown that the current density at the focus is

$$
J_{e}=\frac{\left|\beta_{0}^{3}\right| \gamma_{0}^{3} I_{A}}{\pi z_{e}^{2}}
$$

where $I_{A}=4 \pi \varepsilon_{0} m c^{3} /|q|=17 \mathrm{kA}$ is the Alfvén current [24]. The gain is then

$$
G_{\max }-1=2\left(\frac{1}{3 \pi} \frac{\mu_{0} q^{2}}{m^{2} c^{3}} \frac{1-\beta_{0}}{\left|\beta_{0}\right|} \frac{\mathrm{U}}{\Delta t}\right)^{2 / 3},
$$

independent of the pump wavelength, the electron-beam voltage, and the total current. This result is shown as a red line in Fig. 5. It shows that space charge in the focused electron beam establishes a limit on how long the pulse 
can be. Equivalently, it shows that for sufficiently long pulses or sufficiently high total current, the gain depends only on the peak power $U / \Delta t$ of the pump laser.

In summary, the nonuniform intensity of a realistic optical pulse leads to structure in the spectrum of radiation that is Thomson backscattered from charged particles. This structure is particularly interesting for the possibility it offers for enhanced optical gain on the backscattered radiation and the development of tabletop free-electron lasers. For example, for a $100-\mathrm{kV}, 100-\mathrm{mA}$ beam in the field of a 500-J, 100-ps, 10.054- $\mu \mathrm{m}$ laser pulse focused to a $170-\mu \mathrm{m}$ diameter spot, the Madey theorem predicts a small-signal gain exceeding 100 per pass at a wavelength of $312 \mathrm{~nm}$. The actual gain is almost certainly orders of magnitude larger, but a more sophisticated computation (probably involving numerical simulations) is needed to prove this [31]. Scaling to shorter wavelengths is possible, but the following limitations must be borne in mind. For constant pump laser energy and pulse length, and constant electron-beam brightness and current, the gain falls off roughly with the laser wavelength $\lambda$. This can be compensated by increasing the pulse length or electron-beam current, but the pulse length and current are limited by space-charge defocusing of the electron beam. Larger pump laser energy also increases the gain, but higher electron-beam brightness helps only until space charge dominates the focus. Both the spectral oscillations and the gain should be observable in Thomson backscatter from sufficiently dense, cold plasmas.

\section{ACKNOWLEDGMENTS}

This work was supported by the Medical Free Electron Laser Program of the Department of Defense under Grant No. F49620-01-1-0429.

[1] S. H. Park et al., Nucl. Instrum. Methods Phys. Res., Sect. A 475, 425 (2001).

[2] F. E. Carroll et al., Bull. Am. Phys. Soc. 45, 54 (2000).

[3] I.V. Pogorelsky et al., in Development of HighBrightness Laser Synchrotron Source at BNL ATF2000, Proceedings of the 9th Workshop on Advanced Accelerator Concepts, Santa Fe, NM, 2000, AIP Conf. Proc. No. 569 (AIP, New York, 2001), p. 571.

[4] S.-Y. Chen, A. Maksimchuck, and D. Umstadter, Nature (London) 396, 653 (1998).

[5] R. H. Pantell et al., IEEE J. Quantum Electron. 4, 905 (1968).

[6] J. Gea-Banacloche et al., IEEE J. Quantum Electron. 23, 1558 (1987).
[7] E. Esarey and P. Sprangle, Phys. Rev. A 45, 5872 (1992).

[8] C. A. Brau, Nucl. Instrum. Methods Phys. Res., Sect. A 407, 1 (1998).

[9] D. F. Gordon et al., Nucl. Instrum. Methods Phys. Res., Sect. A 475, 190 (2001).

[10] H.-D. Nuhn, Nucl. Instrum. Methods Phys. Res., Sect. A 445, 149 (2000).

[11] P. Maine et al., IEEE J. Quantum Electron. 24, 398 (1988).

[12] C. H. Boulware and C. A. Brau, in Proceedings of the 24th International Free-Electron Laser Conference, Argonne, IL, 2002, edited by K.-J. Kim, S.V. Milton, and E. Gluskin (Elsevier, Amsterdam, 2003).

[13] A. Gover and P. Sprangle, IEEE J. Quantum Electron. 17, 1196 (1981).

[14] E. Esarey, S. K. Ride, and P. Sprangle, Phys. Rev. E 48, 3003 (1993).

[15] K. Lee et al., Phys. Rev. E 67, 026502 (2003).

[16] C. A. Brau, Free-Electron Lasers (Academic Press, San Diego, 1990), Chap. 5.

[17] C. A. Brau, Modern Problems in Classical Electrodynamics (Oxford University Press, New York, 2003), Chap. 10.

[18] J. D. Jackson, Classical Electrodynamics (John Wiley \& Sons, New York, 1999), 3rd ed., Chap. 14.

[19] C. A. Brau, Modern Problems in Classical Electrodynamics (Oxford University Press, New York, 2003), Chap. 4.

[20] L. D. Landau and E. M. Lifshitz, The Classical Theory of Fields (Pergamon Press, Oxford, 1975), Sec. 47.

[21] C. A. Brau, Free-Electron Lasers (Academic Press, San Diego, 1990), Chap. 3.

[22] J. M. J. Madey, Nuovo Cimento Soc. Ital. Fis., B 50, 64 (1979).

[23] G. F. Carrier, M. Krook, and C. E. Pearson, Functions of a Complex Variable (McGraw Hill, New York, 1966), Chap. 6.

[24] J. D. Lawson, The Physics of Charged-Particle Beams (Oxford University Press, New York, 1988).

[25] C. A. Brau, Free-Electron Lasers (Academic Press, San Diego, 1990), Chap. 4.

[26] B. E. Wyborn, Rutherford Appleton Laboratory Annual Report, 2002/2003.

[27] W. B. Colson, in Classical Free-Electron Laser Theory, edited by W. B. Colson, C. Pellegrini, and A. Renieri, Laser Handbook Vol. 6 (North-Holland, Amsterdam, 1990).

[28] K.-J. Kim, Nucl. Instrum. Methods Phys. Res., Sect. A 250, 396 (1986).

[29] G. Dattoli and A. Renieri, in The Quantum Analysis of the Free Electron Laser, Laser Handbook (Ref. [27]).

[30] C. B. Schroeder, C. Pellegrini, and P. Chen, Phys. Rev. E 64, 056502 (2001).

[31] W. B. Colson, in Classical Free-Electron Laser Theory, Laser Handbook (Ref. [27]). 